\title{
Aspectos Relevantes da Anestesia na Videocirurgia Colorretal
}

\section{Relevants Aspects of Anesthesia in Laparoscopic Colorectal Surgery}

\author{
ADRIANE MARIA GORI PEDROSO ${ }^{1}$; LAURO MARUBAYASHI $^{1}$; REGINAGORI ${ }^{1}$; MIGUEL ANGELOPEDROSO²; \\ RENATOARIONE LUPINACCI ${ }^{2}$ \\ ${ }^{1}$ Serviço de Anestesiologia do Hospital Santa Paula - São Paulo - SP; ${ }^{2}$ Serviço de Cirurgia Geral do Hospital do \\ Servidor Público Estadual de São Paulo - SP.
}

\begin{abstract}
PEDROSO AMG; MARUBAYASHI L; GORI R; PEDROSO MA; LUPINACCI RA. Aspectos Relevantes da Anestesia na Videocirurgia Colorretal. Rev bras Coloproct, 2007;27(3): 284-287.

RESUMO: Os procedimentos cirúrgicos incluindo a videolaparoscopia são realizados atualmente de forma cada vez mais freqüiente, existindo fortes evidências de que sejam acompanhados de uma menor resposta dolorosa, inflamatória, endócrina e metabólica ao trauma operatório quando comparada à cirurgia convencional. Entretanto, algumas de suas características próprias obrigam à uma visão dos aspectos relacionados não apenas à prática cirúrgica, mas também aos procedimentos anestésicos envolvidos, os quais poderão, se inadequados, inviabilizar ou comprometer seriamente a realização da cirurgia pela via laparoscópica.Dentre essas características próprias, destacamos em especial a necessidade do uso prolongado do pneumoperitônio e as freqüentes alterações no posicionamento do paciente. O objetivo do presente trabalho é realizar uma breve revisão dos aspectos fisiológicos presentes, relacionados a cirurgia videolaparoscópica, assim como realizar uma discussão sobre sua influência nos procedimentos anestésicos a serem adotados nestes casos, visando reduzir eventuais efeitos inadequados para o paciente ou para a qualidade do ato operatório.
\end{abstract}

Descritores: Pneumoperitonio, Pressão Intraabdominal (PIA), Videocirurgia Colorretal, Anestesia, Alterações Fisiológica.

\section{PNEUMOPERITÔNIO}

Sabemos que as alterações fisiológicas decorrentes do pneumoperitônio podem resultar em intercorrências clínicas, em especial, quando associadas ao posicionamento dos pacientes durante as videocirurgias colorretais, as quais devem ser minimizadas pelo anestesiologista através de sua técnica anestésica. Estas alterações se devem ao aumento da pressão intraabdominal (PIA), onde o dióxido de carbono (CO2) é o gás mais utilizado e sua eliminação ocorre apenas pelos pulmões. Durante o pneumoperitônio, pode ocorrer um aumento da $\mathrm{PaCO} 2$ (pressão parcial do $\mathrm{CO} 2$ arterial ) em conseqüência de vários fatores como a absorção de $\mathrm{CO} 2$ pela cavidade peritonial, piora na relação ventilação-perfusão através do aparecimento de eventuais "shunts" intrapulmonares, atelectasias resultantes da elevação do diafragma e aumento da pressão intratorácica (decorrentes do aumento da pressão intra-abdominal) ${ }^{2,4,20}$. Eventualmente, estas alterações podem ser minimizadas através da dinâmica ventilatória durante o ato anestésico-cirúrgico ${ }^{4}$. Com relação às alterações cardiovasculares, é sabido que o aumento da PIA resulta em aumento da resistência vascular sistêmica por fatores mecânicos (compressão de artérias e veias abdominais) $)^{3,11,13,19} \mathrm{e}$ fatores humorais como aumento das concentrações de catecolaminas, hormônios do sistema renina-angiotensina-aldosterona (SRAA: conseqüente à redução do fluxo sangüíneo renal) e a vasopressina (neuro-hormônio com liberação aumentada em vigência do pneumoperitônio por estímulos de receptores peritoniais e intracardíacos) ${ }^{6,7,8,9}$. Além do aumento da resistência vascular sistêmica, a elevação

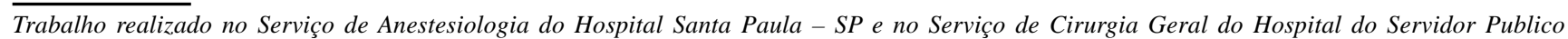
Estadual - SP. 
da PIA contribui para a compressão das artérias e veias abdominais, onde associada ao aumento da pressão intratorácica resulta em diminuição do retorno venoso, e conseqüentemente do débito cardíaco ${ }^{10,11,12,13}$. Em relação à perfusão dos órgãos abdominais, sabemos que esta é proporcional ao aumento da PIA, ocorrendo diminuição do fluxo sangüíneo visceral em aproximadamente: estômago (50\%), duodeno (10\%), jejuno (30\%), cólon (4\%), fígado (35\%) e rins $(25 \%)^{14}$. No que diz respeito à perfusão cerebral, sabemos que esta sofre influências das alterações cardiovasculares, das alterações da $\mathrm{PaCO} 2$, do posicionamento do paciente no intraoperatório e dos aumentos das pressões intraabdomiais e intratorácicas. Como resultado, observamos um aumento da pressão venosa cerebral, e conseqüentemente uma maior incidência de edema cerebral, na maior parte dos casos de caráter transitório, desaparecendo algumas horas após a deflação do pneumoperitônio independentemente de tratamento específico $^{12,22}$. Sabemos que durante o pneumoperitônio e o aumento da PIA ocorre ainda uma redução do retorno venoso, e conseqüente estase venosa em membros inferiores favorecendo a incidência de fenômenos tromboembólicos, os quais podem ser minimizados com uso de perneiras apropriadas e/ou de meias massageadoras de panturrilhas ${ }^{13,21}$.

\section{POSIÇÃO DO PACIENTE}

A dificuldade do acesso manual e instrumentos metálicos para o afastamento de alças intestinais durante as videocirurgias determina com que a variação de posicionamento da mesa cirúrgica seja o principal recurso do cirurgião para a obtenção da mobilidade destas vísceras, visando à obtenção de um campo operatório adequado à realização do ato cirúrgico. No caso específico da videocirurgia colorretal, este aspecto representa um fator essencial devido à necessidade de uma grande amplitude de exposições e procedimentos a serem realizados em todos os quadrantes da cavidade abdominal. Por outro lado, estas variações de posicionamento demandam, freqüentemente uma atenção especial por parte do anestesiologista não apenas em relação à segurança do paciente, devido ao risco de deslocamentos abruptos em virtude de uma fixação inadequada, mas também pelas eventuais alterações fisiológicas potenciais.

Assim sendo, é de extrema importância uma atenção especial à fixação do paciente na mesa cirúr- gica, principalmente da região craniana, ombros e membros inferiores. Além disto, como estas alterações de posicionamento podem requerer a utilização de campos esterelizados mais amplos restringindo por vezes o acesso direto e visual à cabeça do paciente, é necessário um cuidado especial com a monitorização do paciente durante a videocirurgia colorretal, sendo todos os parâmetros relevantes como a freqüência cardíaca, pressão arterial, ritmo cardíaco, freqüência e ritmo pulmonares, saturação de oxigênio, tensão expiratória de $\mathrm{CO} 2$, diurese e gasometria seriada (em casos de pneumopatias e/ou procedimentos por tempo prolon(ado) $)^{16,17}$.

Através da monitorização mínima adequada, o anestesiologista terá condições de suspeitar das graves complicações pelo pneumoperitônio como embolia gasosa, pneumotórax, pneumomediastino, insuflação extra peritonial, enfisema subcutanêo e colapso cardíaco ${ }^{18}$.

\section{ROTINAS ANESTÉSICAS ESPECÍFICAS EM VIDEOCIRURGIA COLORRETAL}

Baseado no acima exposto, desenvolvemos hoje em nosso Serviço uma rotina especifica a ser aplicada em pacientes que serão submetidos a videocirurgia colorretal, a qual contempla as seguintes etapas:

\section{a. Avaliação Pré-Operatória}

Considerando os aspectos fisiológicos acima expostos, estes pacientes deverão submeter-se a uma criteriosa avaliação clínica pré-operatória, incluindo rigorosa investigação de acidentes vasculares cerebrais prévios, fenômenos tromboembólicos, avaliação das funções cardíaca, pulmonar, hepática e renal. Nos casos de pacientes portadores de patologias que incluam disfunções destes órgãos, na avaliação deverá constar exames específicos para tais distúrbios.

\section{b. Técnica Anestésica Utilizada}

Em relação a melhor técnica anestésica para videocirurgia colorretal, ou seja, a mais indicada (seja geral balanceada, geral associada a bloqueios) será a que oferecer as melhores condições para se desenvolver a técnica cirúrgica e minimizar os efeitos fisiológicos causados pelo aumento da PIA durante o pneumoperitônio.

Em nosso serviço atualmente submetemos os pacientes à anestesia geral balanceada ( endovenosa + inalatória) e para intubação orotraqueal realizamos a 
seqüência rápida : pré oxigenação a 100\% em respiração espontânea, administração do indutor + opióide seguidos de substância curarizante despolarizante ( succinilcolina ) ou curarizante de início de ação rápida. Frente a isso, evitamos a ventilação manual prévia à intubação e na sequiência após instituída a ventilação mecânica, realizamos a passagem de sonda nasogástrica.

Esta intubação em seqüência rápida tem se mostrado de grande valor no sentido de reduzir um grande problema na videocirurgia colorretal, representado pela distensão de alças de intestino delgado, as quais por vezes restringem de forma importante a visibilidade do campo operatório podendo mesmo inviabilizar o acesso videolaparoscópico. Baseado em nossa experiência, verificamos que evitando-se a distensão gasosa gástrica, conseguimos menor distensão das alças intestinais, e consequientemente maior adinamia das mesmas. Tal fato associado a excelente curarização e plano anestésico adequado, facilita em muito o desenvolver da técnica cirúrgica e diminui a intensidade das alterações pós pneumoperitônio. Atualmente tem sido raro o uso de escopolamina para obter adinamia das alças, exceto em casos eventuais onde haja um componente semi- obstrutivo.

Em relação a hidratação endovenosa, realizamos a reposição do jejum (lembrando do tipo de preparo) e possíveis perdas do intra-operatório ( em média $8-10 \mathrm{ml} / \mathrm{kg} / \mathrm{h}$ de cristalóides), considerando que as alterações causadas pelo pneumoperitonio ( diminuição da perfusão / compressão de parênquima renal = ativação SRAA) podem resultar em baixo ou ausente débito urinário, independentemente do grau de hidratação. No caso de hiper-hidratação com cristalóides, pode ocorrer certo grau de edema em alças intestinais (principalmente da camada serosa) o que aumentaria a incidência de lesão destas alças através do pinçamento e/ou complicações com anastomoses das mesmas.
Como citado anteriormente, $\mathrm{o}$ aumento da pressão venosa cerebral resultante do aumento da PIA e pressão intratorácica, associado muitas vezes à posição de céfalodeclive prolongada, poderá ocasionar diversos graus de edema cerebral, o qual tem sido muito minimizado em nossos pacientes através do ajuste da mecânica ventilatória no intra-operatório, optando por volume corrente menor e alta frequiência respiratória associada a menor pressão intratorácica possível.

Ainda relacionado ao intra-operatório devemos estar atentos a situações de alterações hidroeletrolíticas, do equilíbrio ácido - básico (por jejum prolongado, preparos de cólon e outros) podendo resultar em estados de acidose metabólica, comprometendo a ação dos curares.

Paciente portador de patologia pulmonar, porém em condições de submeter-se a vídeolaparoscopia, e nos casos de edema cerebral significativo, poderá ter indicação de ventilação mecânica por tempo mais prolongado no pós- operatório imediato, quando comparado com outros pacientes.

Como terapêutica preventiva de fenômenos tromboembólicos, adotamos o uso de perneiras apropriadas no intra-operatório, deambulação precoce e em casos de patologias oncológicas e/ou antecedentes de tromboembolismo, o uso de anticoagulantes subcutâneo no pós-operatório imediato, embora suscetível de questionamento. Para prevenção de náuseas e vômitos utilizamos dexametazona e ondansetrona de rotina logo após a indução anestésica e mantemos a ondansetrona no pós-operatório.

Para analgesia pós-cirúrgica, seguimos os mesmos critérios utilizados em cirurgias pela técnica convencional, associando antiinflamatórios não hormonais (cetoprofeno, tenoxican, dipirona) se não houver contra indicação e opióides (nalbufina, meperidina, morfina) conforme indicação.

\footnotetext{
ABSTRACT: Nowadays, compared to conventional surgery, the increased use of the video-laparoscopic approach in surgery is based on strong evidences of decreased pain, inflammatory, endocrine and metabolic patient response to surgical trauma. However, considering some particularities of its own, it is important to acknowledge aspects of not only the surgical procedure itself, but also of the anesthetic procedures carried out on the patient, which, if inadequately conducted, could seriously jeopardize or compromise the success of the video-laparoscopic surgical intervention. Among these particularities, we would like to specially enhance the need for extended pneumoperitonium applications, as well as the frequent alterations that occur during patient positioning. The main target of this current report, focusing on the reduction of eventual unwanted side effects for the patient or of the quality of the operation, is to briefly review the physiological aspects involved in video-laparoscopic surgery, as well as to discuss their influences on the anesthetic procedures conducted in theses cases.
}

Key Words: pneumoperitonium, intra-abdominal pressure (IAP), laparoscopic colorectal surgery, anesthesia, physiological alterations. 


\section{REFERÊNCIAS}

1. Ganem E.M. - Anestesia para laparoscopia - Atualização em Anestesiologia Vol IX / Saesp

2. Rademaker BM, Bannenberg JJ, Kalkman CJ et al. Effects of pneumoperitoneum with helium on hemordynamics and oxygen transport: a comparison with carbon dioxide. J Laparoendosc Surg 1995;5;15-20.

3. Badner NH. Anesthesia for minimally invasive surgery. Can J Anaesth 1999;46:R101-10

4. Wahba RW,Mamazza J. Ventilatory requirements during laparoscopic cholecystectomy.Can J Anaesth 1993;40:206210.

5. Razvi HA, Fields D, Vargas JC et al. Oliguria during laparoscopic surgery:evidence for direct renal parenchymal compression as an etiologic factor.J Endourol 1996;10:1-4.

6. Harman PK, Kron IL, Mclachlan HD et al. Elevated intra-abdominal pressure and renal function.Ann Surg 1982;196:594-597.

7. McDougall EM, Monk TG, Wolf JS et al.The effect of prolonged pneumoperitoneum on renal function in an animal model.J Am Coll Surg 1996;182:317-328.

8. Punnonen R, Viinamaki O. Vasopressin release during laparoscopy role of intra-abdominal pressure.Lancet 1982;1:175-176.

9. Dunn MD, McDougall EM. Renal physiology.Laparoiscopic considerations.Revew Yale J Biol Med.1998 NovDec;71(6):551-78.

10. Cunningham Aj. Anesthetic implications of laparoscopic surgery. Surg Endosc. 1998 Feb(2):93-4.

11. Joris JL, Noirot DP, Legrand MJ et al. Hemodynamic changes during laparoscopic cholecistectomy. Anesth Analg 1993;76:1067-1071.

12. O'Malley $\mathrm{C}<$ Cunningham Aj, Physiologic changes during laparoscopic. Anesthesiol Clin North America 2001;19:1-19.

13. Takata M, Wise RA, Robotham JL. Effects of abdominal pressure on venous return:abdominal vascular zone conditions. J Appl Physiol 1990;69:19611972.

14. Schilling MK, Redaelli C, Krahenbuhl L et al. Splanchnic microcirculatory changes during CO2 laparoscopy. J AM Coll Surg 1997;184:378-382.
15. Morrison CA, Schreiber MA, Olsen SB et al. Femoral venous flow dynamics during intraperitoneal and preperitoneal laparoscopic insufflation. Surg Endosc 1998;12:1213-1216.

16. Posso IP. Anestesia para laparoscopia e histeroscopia. In Yamashita AM, Takaoka f, Auler JR JOC, Iwata NM. Anestesiologia SAESP 5 ${ }^{\text {a }}$ ED; São Paulo,Editora Atheneu 2001;731-743.

17. Torres HO, Nunes CEL, Araújo Neto JP. Anestesia em cirurgia vídeo-laparoscópica. Ver Bras Anesthesiol 1995;45:21-32.

18. Browne J, Murphy D, Shorten G. Pneumomediastinum,pneumothorax and subcutaneous emphysema complicating MIS HERNIORRHAPHY. Can J Anaesth 2000;47(1):69-72.

19. Westerband A, Van De Water J, Amzallag M, Lebowitz PW, Nwasokwa ON, Chardayone R, Abou-Taleb A, Wang X, Wise L. Cardiovascular changes during laparoscopic cholecystectomy.PMID:1448734[PubMed-indexed for MEDLINE]

20. Horvath KD, Whelan RL, Lier B, Viscomi S, Barry L, Buck K, Bessler M. The effects of elevated intraabdominal pressure, hypercarbia and positioning on the hemodinamic responses to laparoscopic colectomy in pigs.

21. Millard JA. , Hill BB. , Cook PS. Et al. Intermitent sequential pneumatic compression in prevention of venous stasis associated with pneumoperitoneum during laparoscopic cholecistectomy. Arch Surg 1993; 128: 914-19.

22. Halverson AL, Barrett WL, Iglesias AR et al. Decreased cerebrospinal fluid absorption during abdominal insufflation. Surg Endosc 1999; 13: 797-800.

Endereço para correspondência: ADRIANE MARIA GORI PEDROSO

Rua Velho Realejo N $^{\circ} 261$

Condomínio Monte Belo

Cep: 13.323-670

Salto SP

Fax: (011) 4602-2567

E-mail: pedroso.gori@uol.com.br 